\title{
Markers of vitamin B12 status in relation to cerebrospinal fluid biomarkers and cognitive performance
}

\author{
$\underline{\text { Babak Hooshmand }}^{1,2}$, Franziska Koch ${ }^{2}$, Patrik Fissler ${ }^{3}$, Markus Otto $^{2}$, Hayrettin Tumani ${ }^{2}$, \\ Miia Kivipelto ${ }^{1}$ and Christine von Arnim \\ ${ }^{1}$ Karolinska Institute, Stockholm, Sweden, \\ ${ }^{2}$ Ulm University Hospital, Ulm, Germany, \\ ${ }^{3}$ Ulm University Hospital, Ulm, Gibraltar and \\ ${ }^{4}$ Ulm University Hospital, UlmUlm, Germany
}

\section{Abstract}

Introduction: The association between markers of vitamin B12 status and cerebrospinal fluid (CSF) biomarkers of Alzheimer's type dementia which precede cognitive impairment has been investigated by only a few small studies and the results have been inconsistent.

Aim: To investigate the associations of vitamin B12 related markers (vitamin B12, holotranscobalamin (HoloTC), homocysteine (tHcy), and methylmalonic acid (MMA)) with CSF total tau, Amyloid- $\beta 42$ (A $\beta 42$ ) and cognitive performance.

Methods: Data included 462 patients aged 40-94 years referred to the Memory Clinic at the Ulm University Hospital, Ulm, Germany between December 2009 and August 2015. Vitamin B12 and HoloTC were measured via chemiluminescence microparticle immunoassay, tHcy via chemiluminescence immunoassay and MMA via liquid chromatography mass specterometry. CERAD battery was used to examine the cognitive status, and different domains were derived. Regression models were used to investigate the associations.

Results: After adjusting for age, sex, creatinine levels and APOE€4 status, higher values of vitamin B12 and lower values of MMA were associated with lower concentrations of CSF total-tau: the odds ratios (ORs) $(95 \%$ confidence intervals $(\mathrm{CI}))$ in a binary logistic regression analysis investigating the associations with total tau cut-off of $400 \mathrm{pg} / \mathrm{ml}$ were $0.39(0.15-0.99)$ and 5.60 (1.93-16.26) for the highest quartile of B12 and MMA compared to the lowest, respectively. Furthermore, HoloTC, MMA, and tHcy were associated with several cognitive domains such as episodic memory and executive functioning. No relationships were found with $A \beta 42$.

Conclusions: Vitamin $\mathrm{B}_{12}$ and its related markers may be independent predictors of CSF biomarkers of Alzheimer's disease and cognitive status. Randomized controlled trials are needed to determine the importance of vitamin B12 supplementation on slowing structural brain changes and cognitive decline in older adults.

\section{Conflict of Interest}

There is no conflict of interest. 